Internist 2021 · 62:106-110

https://doi.org/10.1007/s00108-020-00937-3

Angenommen: 16. Dezember 2020

Online publiziert: 23. Dezember 2020

(c) Springer Medizin Verlag GmbH, ein Teil von

Springer Nature 2020

\section{Redaktion}

C. Bokemeyer, Hamburg

M. Hallek, Köln

C. Jacobshagen, Karlsruhe

W. Lehmacher, Köln

U. Müller-Ladner, Bad Nauheim

H. Wedemeyer, Hannover

M. Wehling, Mannheim

\section{Originalliteratur}

Voysey M, Clemens SAC, Madhi SA et al on behalf of the Oxford COVID Vaccine Trial Group (2020) Safety and efficacy of the ChAdOx1 nCoV-19 vaccine (AZD1222) against SARSCoV-2: an interim analysis of four randomised controlled trials in Brazil, South Africa, and the UK. Lancet. https://doi.org/10.1016/S01406736(20)32661-1

Polack FP, Thomas SJ, Kitchin N et al for the C4591001 Clinical Trial Group (2020) Safety and efficacy of the BNT162b2 mRNA Covid19 vaccine. $N$ Engl J Med. https://doi.org/10. 1056/NEJMoa2034577

Seit der ersten beschriebenen Infektion mit dem „severe acute respiratory syndrome coronavirus 2" (SARS-CoV-2) im Dezember 2019 hat sich die „coronavirus disease 2019“ (COVID-19) zu einer Pandemie entwickelt, deren Symptomatik vom asymptomatischen Verlauf bis hin zum akuten Lungen- und Multiorganversagen und Tod reicht. Hoffnung, die Pandemie in den Griff zu bekommen, bringen die neu entwickelten, kurz vor der Zulassung stehenden (Stand 21.12.2020) Impfstoffe.

Derzeit sind 52 Impfstoffe in der klinischen Prüfung, die sich hinsichtlich der Wirkweise teils deutlich unterscheiden. So nutzt AZD1222 ein Schimpansenadenovirus als Vektor für SARS-CoV-2Proteine, Sputnik V [5] zwei unterschiedliche Adenoviren als Vektoren, bei der ersten Impfung rAd26 und bei der zweiten rAd5. Der NVX-CoV2373-Impfstoff

\author{
M. Augustin ${ }^{1} \cdot$ M. Hallek' $\cdot$ S. Nitschmann ${ }^{2}$ \\ 'Klinik I für Innere Medizin, Universitätsklinikum Köln (AöR), Köln, Deutschland \\ ${ }^{2}$ Lippetal, Deutschland
}

\title{
Impfstoffentwicklung zur Prävention von COVID-19
}

[4] ist ein rekombinanter Nanopartikelimpfstoff, der aus dem SARS-CoV-2Wildtyp-Spike hergestellt wurde, während mRNA-1273 [3] die Boten-RNA des Spike-Proteins von SARS-CoV-2 als Induktor nutzt und BNT162b2 ein lipidnanopartikelformulierter nukleosidmodifizierter RNA-Impfstoff ist, der das SARSCoV-2-Spike-Protein in voller Länge codiert.

Ziel der im Folgenden vorgestellten Studien war es, die Wirksamkeit einer Impfung von Probanden bezüglich der Prävention einer COVID-19-Infektion $\mathrm{zu}$ verifizieren.

\section{Zusammenfassung der Studien}

\section{S. Nitschmann}

Lippetal, Deutschland

Um einen aktuellen Überblick zu geben, stellen wir hier nur die Ergebnisse der Studien vor, die Daten von mehr als 10.000 Probanden umfassen (Stand 21.12.2020).

\section{Oxford COVID Vaccine Trial Group}

\section{Studiendesign}

Laufende einfachblinde, kontrollierte, randomisierte Multicenterimpfstudie mit einem an der Universität Oxford entwickelten replikationsdefizienten adenoviralen Schimpansenvektor, der das SARS-CoV-2-Oberflächenglykoprotein-Antigen (Spike-Protein; nCoV-19) enthält.

\section{Einschlusskriterien}

- Über 18-jährige Probanden mit berufsbedingt erhöhtem Infektionsrisiko (COV002)

- Über 18-jährige Probanden aus Berufen des Gesundheitssektors (COV003)

\section{Endpunkte Primär:}

- Nachweis einer symptomatischen COVID-19-Infektion (Fieber $\geq 37,8^{\circ} \mathrm{C}$, Kurzatmigkeit, Geruchs-/ Geschmacksverlust) mehr als 14 Tage nach der zweiten Impfung

Sekundär:

- Impfstoffsicherheit

\section{Methodik}

In die hier vorgestellte gepoolte Zwischenanalyse gehen die Daten aus den Phase-II/III-Studien aus Großbritannien (COV002) und Brasilien (COV003) bis zum 04.11.2020 ein. Mittlerweile gibt es weitere Studien in Südafrika (dop-

Take home message
Zusammengefasst stellen die hier
vorgestellten Impfstoffkandidaten AZD1222
(AstraZeneca/University of Oxford)
und BNT162b2 (BioNTech/Pfizer) nach
derzeitigem Kenntnisstand sichere und
effektive Impfstoffe zur Prävention von
COVID-19 dar. Die Zulassungskriterien der
Food and Drug Administration (FDA; [1]) für
die Zulassung von Impfstoffen zur Prävention
von COVID-19 (Effektivität > 50\%) werden
von beiden Kandidaten erfüllt.




\begin{tabular}{|c|c|c|c|c|c|c|}
\hline \multirow{2}{*}{$\begin{array}{l}\text { Probanden- } \\
\text { gruppen }\end{array}$} & \multirow{2}{*}{$\begin{array}{l}\text { COVID-19- } \\
\text { Infektion } \\
(n)\end{array}$} & \multicolumn{2}{|c|}{ ChAdOx1 nCoV-19 } & \multicolumn{2}{|l|}{ MenACWY } & \multirow{2}{*}{$\begin{array}{l}\text { Impfeffektivität (und } \\
95-\mathrm{KI} \text { ) }\end{array}$} \\
\hline & & $n / N(\%)$ & $\begin{array}{l}\text { Inzidenz/1000 Personen- } \\
\text { jahre }\end{array}$ & $n / N(\%)$ & $\begin{array}{l}\text { Inzidenz/1000 Personen- } \\
\text { jahre }\end{array}$ & \\
\hline COV002 & 86 & $18 / 3744(0,5 \%)$ & 38,6 & $68 / 3804(1,8 \%)$ & 145,7 & $73,5 \%(55-84,2)$ \\
\hline LD/SD & 33 & $3 / 1367(0,2 \%)$ & 14,9 & $30 / 1374(2,2 \%)$ & 150,2 & $90,0 \%(67,4-97,0)$ \\
\hline SD/SD & 53 & $15 / 2377(0,6 \%)$ & 56,4 & $38 / 2430(1,6 \%)$ & 142,4 & $60,3 \%(28,0-78,2)$ \\
\hline $\begin{array}{l}\text { Asymptoma- } \\
\text { tisch }\end{array}$ & 69 & $29 / 3288(0,9 \%)$ & 69,8 & $40 / 3350(1,2 \%)$ & 96,0 & $27,3 \%(-17,2$ bis 54,9$)$ \\
\hline COV003 & 45 & $12 / 2063(0,6 \%)$ & 56,2 & $33 / 2025(1,6 \%)$ & 157,0 & $64,2 \%(30,7-81,5)$ \\
\hline
\end{tabular}

pelblind) und Kenia, deren Ergebnisse nicht in diese Analyse eingehen.

Alle Probanden waren angehalten, sich bei potenziellen COVID-19-Symptomen $\mathrm{zu}$ melden und wurden dann klinisch untersucht. Zudem wurde ein Nukleinsäureamplifikationstest (NAAT) durchgeführt und Laborparameter bestimmt. Die Ergebnisse zusätzlich durchgeführter externer Abstriche gingen in dieStudienauswertung ein. Die COV002Probanden erhielten zusätzlich Abstrichsets zum wöchentlichen Selbsttest, um auch asymptomatische COVID-19-Patienten zu detektieren.

COV002 Seit dem 28.05.2020 laufende Studie an 19 Zentren in Großbritannien mit 10.673 gesunden Probanden mit erhöhtem Infektionsrisiko. Die Probanden erhielten 1:1 randomisiert entweder eine erste Low-dose(LD)-Impfung mit $2,2 \cdot 10^{10}$ Viruseinheiten ChAdOx1 nCoV-19 und anschließend die Standarddosis (SD) von $5 \cdot 10^{10}$ Viruseinheiten ChAdOx1 nCoV-19 oder eine 2-malige Impfung mit der SD oder mit MenACWY (einem Meningokokkenimpfstoff; Kontrollgruppe).

COV003 Seit dem 23.06.2020 laufende Studie an 6 Zentren in Brasilien mit 4088 gesunden Probanden mit erhöhtem Infektionsrisiko. Die Probanden erhielten 1:1 randomisiert entweder eine erste LD-Impfung mit $2,2 \cdot 10^{10}$ Viruseinheiten ChAdOx1 nCoV-19 und anschließend die SD oder eine 2-malige Impfung mit der SD oder mit MenACWY (erste Dosis) und Kochsalz (zweite Dosis; Kontrollgruppe).

\section{Ergebnisse}

In die hier vorgestellte Effektivitätsanalyse gingen 7548 der 10.673 COV002-Probanden und 4088 der 10.002 COV003Probanden ein. Von diesen erhielten 5807 die Verum- und 5829 die Kontrollimpfung. Der allergrößte Teil der Probanden $(>86 \%)$ war zwischen 18 und 55 Jahren alt, $60,5 \%$ waren Frauen. Die Zeitspanne zwischen den Impfungen variierte zwischen 36 Tagen bei COV003 und 84 Tagen bei COV002 bei der LD/SDKombination. Bei Studienbeginn seropositiv waren $1,3 \%$ der COV002- und 2,3\% der COV003-Kohorte.

Insgesamt wurde bei 30 der 5807 Verum- und bei 101 der 5829 Kontrollprobanden eine symptomatische COVID19-Infektion nachgewiesen $(0,5 \%$ vs. 1,7\%; 44,1 bzw. 149,2 Ereignisse pro 1000 Personenjahre), was einer Impfeffektivität von 70,4\% entspricht (• Tab. 1).

Positive Nasenabstriche wurden bei 68 der 5807 Verum- und bei 153 der 5829 Kontrollprobanden gewonnen ( $1,2 \%$ vs. $2,6 \%$; 68 bzw. 153 Ereignisse pro 1000 Personenjahren), was einer Impfeffektivität von 55,7\% entspricht.

Im gesamten Probandenkollektiv erkrankten nur wenige Probanden schwer an COVID-19: keiner der $12.021 \mathrm{ChA}$ dOx1-nCoV-19-Probanden und nur 2 der 11.724 Kontrollprobanden (einer mehr als 14 Tage nach der zweiten Dosis und einer mehr als 21 Tage nach der ersten und weniger als 14 Tage nach der zweiten Dosis). Krankenhausaufnahmen aufgrund von COVID-19 waren bei 2 der ChAdOx1-nCoV-19-Probanden weniger als 21 Tage nach der ersten Dosis und bei insgesamt 16 der Kontrollprobanden indiziert ( 6 weniger als 21 Tage nach der ersten Dosis, 5 mehr als 14 Tage nach der zweiten Dosis und $5 \mathrm{mehr}$ als 21 Tage nach der ersten und weniger als 14 Tage nach der zweiten Dosis).

Die Sicherheitsanalyse ergab ein gutes Sicherheitsprofil für ChAdOx $1 \mathrm{nCoV}$ 19: 79 Probanden entwickelten schwere Impfreaktionen verglichen mit 89 Probanden der Kontrollgruppe.

\section{BNT162b2-mRNA-COVID-19- Vakzine}

\section{Studiendesign}

Laufende beobachterblinde, placebokontrollierte, randomisierte Multicenterimpfstudie an 152 Zentren überwiegend in den USA (130 Zentren), in Südamerika, Südafrika, Deutschland und der Türkei mit einem lipidnanopartikelformulierten nukleosidmodifizierten RNAImpfstoff, der für ein SARS-CoV-2Spike-Protein in voller Länge codiert.

\section{Einschlusskriterien}

- Gesunde, über 16-jährige Probanden

\section{Ausschlusskriterien}

- Anamnestische COVID-19-Infektion

- Autoimmuntherapie bzw. Immunschwäche

\section{Endpunkte Primär:}

- Impfstoffsicherheit: lokale oder systemische unerwünschte Ereignisse, Einnahme von Antipyretika oder Schmerzmitteln binnen 7 Tagen nach Impfung

- Nachweis einer laborbestätigten COVID-19-Infektion frühestens 7 Tage nach der zweiten Impfdosis bei bis dahin seronegativen Probanden 
- Nachweis einer laborbestätigten COVID-19-Infektion frühestens 7 Tage nach der zweiten Impfdosis unabhängig von einer eventuellen SARS-CoV-2-Infektion

\section{Sekundär:}

- Wirksamkeit gegen schwere COVID19

\section{Methodik}

Die Probanden wurden 1:1 auf 2 Dosen à $30 \mu \mathrm{g}(0,3 \mathrm{ml})$ des BNT162b2-Impfstoffs im Abstand von 21 Tagen oder 2 entsprechende Placeboimpfdosen randomisiert. Die Probanden sollten über 7 Tage ein elektronisches Tagebuch führen, anschließend sollten sie unerwünschte Nebenwirkungen bis 6 Monate nach der zweiten Dosis melden.

Für eine bestätigte COVID-19-Infektion musste mindestens eines der folgenden Symptome vorhanden sein: Fieber, neuer/verstärkter Husten, neue/ verstärkte Atemnot, Schüttelfrost, neue Muskelschmerzen, neuer Geschmacksoder Geruchsverlust, Halsschmerzen, Durchfall oder Erbrechen. Weiterhin musste eine Atemprobe während der symptomatischen Periode ( \pm 4 Tage) mittels Nukleinsäureamplifikation positiv für SARS-CoV-2 sein.

\section{Ergebnisse}

Es werden die Daten vorgestellt, die denen im Zulassungsantrag entsprechen. In die hier vorgestellte Interimsanalyse gingen die Daten von 43.448 geimpften Probanden während der Phase-I- bis PhaseIII-Studie ein. Von diesen Probanden erhielten 21.720 zwei Dosen à $30 \mu \mathrm{g}$ des BNT162b2-Impfstoffs im Abstand von 21 Tagen und 21.728 zwei entsprechende Placeboimpfdosen. Die von der Food and Drug Administration (FDA) definierte Hauptsicherheitsgruppe, in der die Follow-up-Zeit $\geq 2$ Monate betrug, umfasste 37.706 Probanden, von diesen erhielten 18.860 BNT162b2 und 18.846 Placebo.

Das Geschlechterverhältnis war nahezu ausgeglichen (50,6\% vs. 49,4\%), das Durchschnittsalter betrug 52 Jahre (16-91 Jahre) und bei etwas mehr als einem Drittel der Probanden betrug der Body-Mass-Index $>35 \mathrm{~kg} / \mathrm{m}^{2}$.
Bei 8 der seronegativen BNT162b2und bei 162 der seronegativen Placeboprobanden wurde eine COVID-19Infektion mindestens 7 Tage nach der zweiten Impfdosis nachgewiesen, was einer Impfeffektivität von 95\% (95\%Konfidenzintervall 90,3-97,6\%) entspricht.

Unabhängig von einer stattgehabten Infektion wurde bei 9 der BNT162b2und bei 169 der Placeboprobanden eine COVID-19-Infektion mindestens 7 Tage nach der zweiten Impfdosis nachgewiesen, was einer Impfeffektivität von 94,6\% (95\%-Konfidenzintervall 89,9-97,3\%) entspricht. Subgruppenanalysen ergaben, dass diese Ergebnisse unabhängig von Probandenalter, Geschlecht, ethnischer Zugehörigkeit, Fettsucht und medizinischen Begleitfaktoren konsistent waren.

Eine schwere COVID-19-Infektion trat bei einem der BNT162b2- und bei 9 der Placeboprobanden auf.

Die Sicherheitsanalyse über den 2-monatigen Follow-up-Zeitraum zeigte ein gutes Sicherheitsprofil für BNT162b2: Es traten kurzfristige leichte bis mittelschwere Schmerzen an der Injektionsstelle, Müdigkeit und Kopfschmerzen auf. Systemische Nebenwirkungen wurden häufiger von jüngeren als von älteren Probanden beschrieben. Insgesamt wurden von $27 \%$ der BNT162b2- und $21 \%$ der Placeboprobanden Nebenwirkungen berichtet. Eine Lymphadenopathie entwickelten 0,3\% der BNT162b2- und $<0,1 \%$ der Placeboprobanden.

\section{Kommentar}

\section{Augustin, M. Hallek}

Klinik I für Innere Medizin, AG Lehmann, Universitätsklinikum Köln (AöR), Köln, Deutschland

Mit aktuell 52 Impfstoffkandidaten [2] in der klinischen Prüfung ist der Anfang vom Ende der SARS-CoV-2-Pandemie eingeläutet (Stand 21.12.2020). Zielstruktur der meisten Impfungen ist das Spike-Protein Glykoprotein S, das nach Bindung an den Angiotensin-converting-enzyme-2(ACE2)-Rezeptor für den Zelleintritt von SARS-CoV-2 verantwortlich ist. Gegen das Glykoprotein S werden nach erfolgreicher Impfung im besten Fall humorale und zelluläre Immunantworten in Gang gesetzt. Auf die in der klinischen Prüfung am weitesten fortgeschrittenen Kandidaten der zwei vielversprechendsten Impfstoffklassen werden wir hier vorrangig eingehen:

- AZD1222 (AstraZeneca/University of Oxford) als Vertreter nichtreplizierender Vektorimpfstoffe

- BNT162b2 (BioNTech/Pfizer) als Vertreter der MessengerRibonukleinsäure(mRNA)-Impfstof$\mathrm{fe}$

In beiden Fällen liegen methodisch korrekte, placebokontrollierte, 1:1 randomisierte sowie verblindete klinische PhaseII/III-Studien zur Evaluation der Sicherheit und Effektivität einer 2-zeitigen Verabreichung (Intervall 28 Tage vs. 21 Tage) des jeweiligen Impfstoffkandidaten vor.

\section{AZD1222}

Bei Vektorimpfstoffen werden abgeschwächte, meist adenovirale Vektoren als Transportmittel für die Zielstruktur in den Körper verwendet. Eine besondere Herausforderung bei vektorbasierten Impfstoffen ist die schnell auftretende Vektorimmunität und somit die Auswahl des viralen Vektors. Wird nämlich der idente virale Vektor zu zwei Impfzeitpunkten verwendet, kann es sein, dass die zweite Impfung weniger wirksam ist als die erste. Um dieses Problem zu umgehen, wurde bei AZD1222 ein adenoviraler, dem Menschen nicht bekannter Schimpansenvektor ChAdOx1 verwendet.

Um die Sicherheit und Wirksamkeit in verschiedenen Ethnizitäten nachzuweisen, wurden 7548 Probanden in Großbritannien (COV002) sowie 4088 Probanden in Brasilien (COV003) in die Zwischenanalyse eingeschlossen (insgesamt 11.636 Probanden). Während die Effektivitätsanalyse von AZD1222 für alle 11.636 Probanden eine Impfeffektivität von $70,4 \%$ erbrachte, gab es ortsbedingte Unterschiede (Großbritannien/COV002 $73,5 \%$ vs. Brasilien/COV003 64,2\%) sowie dosisbedingte Unterschiede (SD; LD) bei COV002 (SD/SD 60,3\% vs. LD/SD $90,0 \%)$. Jene Probanden, die zum ersten Zeitpunkt die Halbdosis verabreicht 
bekamen, wiesen eine deutlich höhere Impfeffektivität auf als jene Probanden mit zweimaliger Standarddosierung. Gründe hierfür könnten in Veränderungen der oben erwähnten Vektorimmunität oder unterschiedlichen humoralen oder zellulären Immunkapazitäten liegen. Insgesamt konnte AZD1222 angesichts schwerer unerwünschter Ereignisse (SAE) bei 79 Verumprobanden vs. 89 Kontrollprobanden als sicher bewertet werden.

Ein weiterer vektorbasierter Impfstoffkandidat ist Sputnik V, der vom russischen Gamaleya Research Institute [5] entwickelt wurde. Hier wurde zur Minimierung der Vektorimmunität auf zwei verschiedene adenovirale Vektoren gesetzt: zum Zeitpunkt 1 rAd26 und zum Zeitpunkt 2 rAd5 [5]. Die aktuell vorliegenden Daten aus einer offenen, nichtrandomisierten Phase-I/II-Studie mit 76 Probanden erlauben keine valide Antwort auf die Frage nach der Effektivität von Sputnik V - eine randomisierte, verblindete und placebokontrollierte Phase-III-Studie ist ausstehend.

\section{BNT162b2}

Bei der jüngeren Technologie der Ribonukleinsäureimpfung wird dem Körper mittels mRNA ein Bauplan für die Zielstruktur, das SARS-CoV-2-Spike-Protein, bereitgestellt. Eine multizentrische, methodisch korrekte Studie zur Evaluation der Sicherheit und Effektivität von BNT162b2 (BioNTech/Pfizer) an insgesamt 43.448 Probanden (21.720 Verumprobanden vs. 21.728 Kontrollprobanden) erzielte eine Impfeffektivität von $95 \%$. Während die Geschlechtsund Altersverteilung in der Studienpopulation ausgeglichen war (Geschlecht: 50,6\% Männer vs. 49,4\% Frauen; Alter: 16-55 Jahre 57,8\% vs. > 55 Jahre 42,2\%), wurden mehrheitlich weiße $(82,9 \%)$ USStaatsbürger (76,7\%) eingeschlossen. Interessanterweise zeigten sich in der Subgruppenanalyse der Impfeffektivität keine signifikanten Unterschiede in Bezug auf einzelne Ethnien, Geschlecht, Body-Mass-Index oder Alter.

Hier müssen natürlich die unterschiedlichen Probandenzahlen in den einzelnen Subgruppen berücksichtigt werden. Insgesamt konnte BNT162b2 angesichts schwerer unerwünschter Ereignisse (SAE) bei 0,6\% der Verumprobanden vs. 0,5\% der Kontrollprobanden als sicher bewertet werden.

Weitere mRNA-Impfstoff-Kandidaten sind mRNA-1273 von Moderna [3] und $\mathrm{CVnCoV}$ von CureVac [6]. Hier liegen zum jetzigen Zeitpunkt (Stand 21.12.2020) Daten zur guten Sicherheit aus Phase-I-Studien vor (Preprint von CureVac noch nicht peer-reviewed). Die Impfeffektivität wird aktuell in laufenden Phase-II/III-Studien bewertet.

Im Jahr 2021 werden weltweit voraussichtlich zahlreiche Impfstoffe zur Prävention von COVID-19 zugelassen werden $^{1}$. Diese Vielfalt an Impfstoffen wird benötigt, um eine globale Impfstoffverteilung $\mathrm{zu}$ garantieren. Denn abhängig von den erforderlichen Lagerungs- und Transportauflagen sowie Kosten des jeweiligen Impfstoffs sollte die Impfstoffverteilung fair und adäquat abgestimmt werden. Die Fragen nach der Dauer des humoralen und zellulären Impfschutzes sowie nach dem Erreichen einer sterilen Immunität nach erfolgreicher Impfung bleiben offen.

Die in den vorgestellten Studien nachgewiesenen Effektivitäten der Impfstoffkandidaten AZD1222 (70,4\%) und BNT162b2 (95\%) liegen deutlich über den Zulassungskriterien der FDA [1] sowie über der Gesamteffektivität von $45 \%$ aller Influenzaimpfungen in den USA von 2019 bis 2020 [7]. Es ist außerdem anzumerken, dass - hochgerechnet auf 1000 Personen - eine 95 \%ige Effektivität statistisch 6-mal weniger SARS-CoV-2Infektionen bedeutet als eine Wirksamkeit von 70,4\% (50 vs. 300 SARS-CoV2-positive Personen auf 1000 Personen).

Zusammengefasst stellen die hier vorgestellten Impfstoffkandidaten AZD1222 (AstraZeneca/University of Oxford) sowie BNT162b2 (BioNTech/Pfizer) nach derzeitigem Kenntnisstand sichere und effektive Impfstoffe zur Prävention von

\footnotetext{
1 BNT162b2 (BioNTech/Pfizer) wurde am 02.12.2020 in Großbritannien, am 09.12.2020 in Kanada, am 11.12.2020 in den USA und am 21.12.2020 in Europa für die Impfung zur Prävention von COVID-19 zugelassen. (Stand: 21.12.2020).
}

COVID-19 dar. Die Zulassungskriterien der FDA [1] für die Zulassung von Impfstoffen zur Prävention von COVID-19 (Effektivität $>50 \%$ ) werden von beiden Kandidaten erfüllt.

\section{Korrespondenzadresse}

\section{Augustin}

Klinik I für Innere Medizin, Universitätsklinikum Köln (AöR)

Kerpener Str. 62, 50937 Köln, Deutschland

max.augustin@uk-koeln.de

\section{Einhaltung ethischer Richtlinien}

Interessenkonflikt. M. Augustin, M. Hallek und S. Nitschmann geben an, dass kein Interessenkonflikt besteht.

Für diesen Beitrag wurden von den Autoren keine Studien an Menschen oder Tieren durchgeführt. Für die aufgeführten Studien gelten die jeweils dort angegebenen ethischen Richtlinien.

\section{Literatur}

1. Administration, F.A.D. (2020) Guidance for industry: emergency use authorization for vaccines to prevent COVID-19. https://www.fda.gov/media/ 142749/download (Erstellt: 10.2020) (p. 15). Zugegriffen: 13.12.2020

2. R.D. Blueprint (2020) DRAFT landscape of COVID-19 candidate vaccines. https://www. who.int/publications/m/item/draft-landscapeof-covid-19-candidate-vaccines (Erstellt: 10. Dez. 2020) (World Health Organisation). Zugegriffen: 13.12.2020

3. Jackson LA, Anderson EJ, Rouphael NG et al (2020) An mRNA vaccine against SARS-CoV-2-Preliminary report. N Engl J Med 383:1920-1931. https:// doi.org/10.1056/NEJMoa2022483 (the mRNA1273 Study Group)

4. Keech C, Alber G, Chor I et al (2020) Phase 1-2 trial of a SARS-coV-2 recombinant spike protein nanoparticle vaccine. NEnglJMed 383:2320-2332. https://doi.org/10.1056/NEJMoa2026920

5. Logunov DY, Dolzhikova IV, Zubkova OV et al (2020) Safety and immunogenicity of an rAd26 and rAd5 vector-based heterologous primeboost COVID-19 vaccine in two formulations: two open, non-randomised phase $1 / 2$ studies from Russia. Lancet 396(10255):887-897 (https://www. thelancet.com/action/showPdf?pii=S0140-6736 \%2820\%2931866-3)

6. Kremsner P et al (2020) Phase 1 assessment ofthe safety and immunogenicity of an mRNA-lipid nanoparticle vaccine candidate against SARScoV-2 in human volunteers. medRxiv. https://doi. org/10.1101/2020.11.09.20228551

7. Dawood FS et al (2020) Interim estimates of 2019-20 seasonal influenza vaccine effectiveness-United States, February 2020. MMWRMorb Mortal Wkly Rep 69(7):177-182 
Hier steht eine Anzeige.

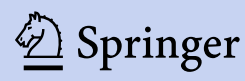

